Abstracta Iranica

Revue bibliographique pour le domaine irano-aryen

Volume 37-38-39 | 2018

Comptes rendus des publications de 2014-2016

\title{
Yury Karev. Samarqand et le Sughd à l'époque 'abbāsside. Histoire politique et sociale
}

\section{Camille Rhoné-Quer}

\section{OpenEdition}

1 Journals

\section{Édition électronique}

URL : http://journals.openedition.org/abstractairanica/47421

DOI : 10.4000/abstractairanica.47421

ISBN : 1961-960X

ISSN : 1961-960X

Éditeur :

CNRS (UMR 7528 Mondes iraniens et indiens), Éditions de l'IFRI

Référence électronique

Camille Rhoné-Quer, «Yury Karev. Samarqand et le Sughd à l'époque 'abbāsside. Histoire politique et sociale », Abstracta Iranica [En ligne], Volume 37-38-39 | 2018, document 11, mis en ligne le 30 décembre 2018, consulté le 28 septembre 2020. URL : http://journals.openedition.org/ abstractairanica/47421; DOI : https://doi.org/10.4000/abstractairanica.47421

Ce document a été généré automatiquement le 28 septembre 2020.

Tous droits réservés 


\title{
Yury Karev. Samarqand et le Sughd à l'époque 'abbāsside. Histoire politique et sociale
}

\author{
Camille Rhoné-Quer
}

\section{RÉFÉRENCE}

Yury Karev. Samarqand et le Sughd à l'époque 'abbāsside. Histoire politique et sociale. Paris : Association pour l'avancement des études iraniennes, 2015, 372 p., ISBN

978-2-910640-41-5 (Cahiers de Studia Iranica 55)

1 Yury Karev fournit une étude détaillée de l'histoire politique et sociale de la Transoxiane du début de la révolution abbasside (747) à l'aube de la période samanide (vers 820). L'intérêt de l'ouvrage réside autant dans son analyse fine des sources archéologiques, numismatiques, historiques - y compris chinoises -, que dans l'exploitation des travaux russophones produits depuis les années 1950. L'auteur s'efforce de reconstituer la chronologie fine des événements. Il analyse par ailleurs les évolutions des élites et l'impact de la colonisation par les conquérants arabomusulmans. Se plaçant dans le cadre d'une approche multiscalaire, l'auteur intègre l'histoire politique des autorités abbassides locales et centrales, des royaumes soumis ou autonomes, et des rivaux extérieurs (Tang ou Turcs).

2 L'ensemble est réparti en 6 chapitres auxquels s'ajoutent une introduction, une conclusion, 3 cartes et deux index. Les chapitres sont organisés de manière chronologique. Le premier chapitre est consacré à « la révolution 'abbāsside et la politique d'Abū Muslim au Māwarā'annahr » (86 pages) et intègre des illustrations sur le programme de construction mené par Abū Muslim en Transoxiane. Le deuxième chapitre (33 pages) porte sur «l'affermissement du pouvoir 'abbāsside » pendant la période entre Abū Muslim et al-Muqanna, que ce soit à travers la politique des gouverneurs ou par le biais d'une politique de contacts diplomatiques qui se seraient 
maintenus avec la Chine au moins jusqu'à la fin du VIIIe siècle. Dans le troisième chapitre est analysé le mouvement messianique d'al-Muqanna' (m. 780), dont le succès serait une conséquence des bouleversements provoqués par les conquêtes arbomusulmanes et l'islamisation. Le quatrième chapitre, relativement court (38 pages), comprend une étude de la politique des gouverneurs dans les dernières décennies du VIIIe siècle ainsi que des relations entre les califes abbassides et les rois locaux d'Orient. L'insurrection de Rāfi' b. Layt (806-809), petit-fils du dernier gouverneur omeyyade du Khorassan, est l'objet du chapitre suivant et est traitée à la manière de celle d'alMuqanna', dans une perspective comparatiste (histoire et chronologie de la révolte, puis étude de ses "forces motrices»), dans un contexte marqué par les tendances centrifuges qui accompagnent la formation de nouvelles élites dirigeantes à la périphérie de l'empire. Le $6^{\mathrm{e}}$ et dernier chapitre (28 pages) prolonge l'étude jusqu'à l'arrivée des Samanides au pouvoir et évoque le rapprochement qui s'opère au début du IXe siècle entre le califat abbasside et les élites locales, y compris l'islamisation de la noblesse.

En dépit de la présence d'hypothèses parfois trop nombreuses et/ou peu convaincantes, ainsi que de l'absence de quelques références historiographiques essentielles, notamment sur le jihad ou sur les Samanides, tout lecteur intéressé par l'histoire des débuts de l'Islam et de la Transoxiane ne pourra que tirer de riches informations ou pistes de réflexion dans cet ouvrage fort dense. Saluons tout particulièrement l'exploitation des sources chinoises ainsi que de la considérable bibliographie russophone.

\section{AUTEURS}

\section{CAMILLE RHONÉ-QUER}

Université d'Aix-Marseille 